\title{
Dose-dependent RNAi-mediated geminivirus resistance in the tropical root crop cassava
}

\author{
Journal Article \\ Author(s): \\ Vanderschuren, Hervé; Alder, Adrian; Zhang, Peng; Gruissem, Wilhelm \\ Publication date: \\ 2009 \\ Permanent link: \\ https://doi.org/10.3929/ethz-b-000021361 \\ Rights / license: \\ In Copyright - Non-Commercial Use Permitted \\ Originally published in: \\ Plant Molecular Biology 70(3), https://doi.org/10.1007/s11103-009-9472-3
}




\title{
Dose-dependent RNAi-mediated geminivirus resistance in the tropical root crop cassava
}

\author{
Hervé Vanderschuren · Adrian Alder · \\ Peng Zhang $\cdot$ Wilhelm Gruissem
}

Received: 7 October 2008/Accepted: 9 February 2009/Published online: 20 March 2009

(C) Springer Science+Business Media B.V. 2009

\begin{abstract}
Cassava mosaic disease is a major constraint for cassava production in Africa, resulting in significant economic losses. We have engineered transgenic cassava with resistance to African cassava mosaic virus (ACMV), by expressing ACMV AC1-homologous hairpin doublestrand RNAs. Transgenic cassava lines with high levels of AC1-homologous small RNAs have ACMV immunity with increasing viral load and different inoculation methods. We report a correlation between the expression of the AC1homologous small RNAs and the ACMV resistance of the transgenic cassava lines. Characterization of the small RNAs revealed that only some of the hairpin-derived small RNAs fall into currently known small interfering RNA classes in plants. The method is scalable to stacking by targeting multiple virus isolates with additional hairpins.
\end{abstract}

Keywords Virus resistance - Cassava .

RNA interference $\cdot$ Hairpin RNA processing

Electronic supplementary material The online version of this article (doi:10.1007/s11103-009-9472-3) contains supplementary material, which is available to authorized users.

H. Vanderschuren $(\bowtie) \cdot$ A. Alder · P. Zhang ·

W. Gruissem ( $\square)$

Department of Biology, Institute of Plant Sciences, ETH Zürich,

Universitätstrasse 2, 8092 Zurich, Switzerland

e-mail: hvanderschuren@ethz.ch

W. Gruissem

e-mail: wgruissem@ethz.ch

P. Zhang $\cdot$ W. Gruissem

Shanghai Center for Cassava Biotechnology, Institute of Plant Physiology and Ecology, Shanghai Institutes for Biological Sciences, Chinese Academy of Sciences, 300 Fenglin Road, Shanghai 200032, China
Cassava mosaic disease (CMD) is caused by several cassava mosaic geminivirus species and is currently the most important disease of cassava in Africa (Legg and Fauquet 2004). Total yield loss due to CMD in Africa was estimated to more than 34 mega tonnes (equivalent to $24 \%$ of total production) for the year 2005 (Legg et al. 2006). The problem that represents geminivirus infection is not restricted to cassava production. Geminivirus diseases have recently spread throughout much of the world in several staple food and cash crops (Boulton 2003; Mansoor et al. 2006). The increasing impact of geminivirus infections on crop yields has emphasized the importance of developing efficient and sustainable geminivirus resistance in the geminivirus-affected crops. Several approaches have been developed to engineer geminivirus resistance in different plant species, especially in model plants for virus studies (Vanderschuren et al. 2007a). So far, most of the reported transgenic plant lines failed to confer high levels of geminivirus resistance or the chosen resistance strategy was effective only in a small subset of transgenic lines (Noris et al. 1996; Chellappan et al. 2004; Zhang et al. 2005; Fuentes et al. 2006; Zrachya et al. 2007; Bonfim et al. 2007; Ribeiro et al. 2007; Vanderschuren et al. 2007b). However, Shepherd et al. (2007) have recently reported delayed symptom development and decreased symptom severity in several transgenic maize lines challenged with Maize streak virus (MSV). Engineering ACMV resistance in cassava using sense RNA and antisense RNA strategies produced lines with varying levels of resistance under high virus inoculation pressure (Chellappan et al. 2004; Zhang et al. 2005).

In recent years, a hairpin dsRNA-based approach has gained interest following its earlier proven efficiency in the RNA virus-model plant system (Smith et al. 2000; Wang 
et al. 2000; Kalantidis et al. 2002). Transient assays using RNA interference (RNAi)-based strategies gave promising initial results in several virus-host systems (Pooggin et al. 2003; Vanitharani et al. 2003). Geminivirus resistance has been reported in transgenic bean and transgenic tomato expressing intron hairpin dsRNA homologous to the geminivirus replication-associated protein sequence (Rep) (Fuentes et al. 2006; Bonfim et al. 2007). In both cases, a high resistance level was only reported for one transgenic line out of all produced. Fuentes et al. (2006) reported that the TYLCV resistant transgenic tomato line had a higher load of hairpin-derived small RNAs when compared to the other transgenic lines. Intron hairpin dsRNA against CP viral sequence did not generate stable resistance in tomato (Zrachya et al. 2007). We recently reported an improved recovery phenotype in ACMV-infected transgenic cassava lines expressing intron hairpin dsRNA homologous to the ACMV common region (CR) (Vanderschuren et al. 2007b). The results that have yet been reported in geminivirus resistance suggest the resistance conferred by the transgenes may not be always sufficiently robust for efficient trait introgression into farmer-preferred cultivars.

We have engineered a CMD-susceptible cassava cultivar (TMS60444) to express a hairpin (hp) dsRNA homologous to a region of the replication-associated protein coding sequence (Rep/ACl) that is highly conserved among bipartite cassava mosaic geminiviruses. The Rep/ $A C l$ protein was targeted because it is essential for geminivirus replication (Hanley-Bowdoin et al. 2000). Several transgenic cassava lines showed ACMV resistance. Based on our findings, we hypothesized that the load of hairpin-derived small RNAs plays a quantitative role in the geminivirus resistance. Analysis of the intron hairpin ds RNA generates several classes of conventional and unconventional small RNAs depending on the intron hairpin segment that is analyzed.

\section{Materials and methods}

Plasmid construction and cassava transformation

The expression binary vector pRNAi-dsAC1 was constructed based on an RNAi plasmid described by Pooggin et al. (2003). The Mungbean yellow mosaic virus (MYMV) sequences were replaced with the ACMV-NOg AC1 sequence from position 1690 to 1844 (GenBank accession AJ427910) in the reverse and the forward orientations. The resulting construct was mobilized into Agrobacterium tumefaciens LBA4404 for transformation of cassava TMS60444 as previously described by Zhang et al. (2000).
Characterization of transgenic lines

Cassava genomic DNA was extracted from freeze-dried leaves according to Soni and Murray (1994). PCR and Southern analyses were carried out following standard protocols. Aliquots of $20 \mu \mathrm{g}$ genomic DNA were digested with HindIII, which cuts the T-DNA once, for Southern Blot analysis. The hybridization probes specific to hygromycin and to ACMV-NOg AC1 gene were DIG-dUTPlabeled by PCR using a PCR DIG probe synthesis kit (Roche Diagnostics GmbH, Germany) according to the manufacturer's instructions. Primers used for PCR are listed in Supplementary Table 1.

Virus bombardment and inoculation assays

Infectious clones of ACMV-NOg were biolistically delivered to 10-week-old cassava plantlets. Each plant was bombarded twice with $0.0625 \mathrm{mg}$ gold particles coated with either $87.5 \mathrm{ng}$ (1st round of infection) or $175 \mathrm{ng}$ (2nd round of infection) of each ACMV component per bombardment as previously described (Zhang et al. 2005). In the 1 st round of infection, the infection was performed on two batches (italic and regular numbers in Table 1, column 1). After bombardment, cassava plants were kept at $28^{\circ} \mathrm{C}$ under $16 \mathrm{~h}$ light and $60 \%$ humidity in the greenhouse.

Infectious clones of ACMV-NOg tandem repeat DNA A and DNA $B$ were transferred to binary vectors (pCAMBIA 1300). pCAMBIA1300-DNA A and pCAMBIA DNA B were mobilized separately to Agrobacterium tumefaciens strain LB4404. Single colonies of Agrobacterium-DNA A and Agrobacterium-DNA B were grown separately on YEB plates supplemented with streptomycin, rifampicin and kanamycin. Equal amounts of each culture were mixed and delivered with a syringe to the wounded apex of cassava plantlets.

Disease symptoms in inoculated and all emerging leaves were recorded during 16 weeks for each plant. The symptom severity on fully expanded leaves was recorded on a scale of $0-4$ as previously described by Fauquet and Fargette (1990).

Virus detection in infected plants

Total DNA was extracted from the pooled 12th (agroinoculation) and 15th (biolistic delivery) leaves of five infected plants grown under greenhouse conditions. Two hundred and fifty ng of DNA was used per PCR reaction. A $770 \mathrm{bp}$ fragment was amplified from the viral AV1 gene for virus detection and quantification. For semi-quantitative PCR, the internal control was the amplification of a $300 \mathrm{bp}$ fragment from a highly conserved region of the ATP 
Table 1 Virus bombardment and inoculation tests

\begin{tabular}{|c|c|c|c|c|c|c|c|c|c|}
\hline Line & $\begin{array}{l}1 \\
\text { Biolistic } \\
\text { delivery } \\
(350 \mathrm{ng} / \text { plant })\end{array}$ & $\begin{array}{l}2 \\
\text { Infection } \\
\text { rate }(\%)\end{array}$ & $\begin{array}{l}3 \\
\text { Total }\end{array}$ & $\begin{array}{l}4 \\
\text { Biolistic } \\
\text { delivery } \\
\text { (700 ng/plant) }\end{array}$ & $\begin{array}{l}5 \\
\text { Infection } \\
\text { rate }(\%)\end{array}$ & $\begin{array}{l}6 \\
\text { Total }\end{array}$ & $\begin{array}{l}7 \\
\text { Agro- } \\
\text { inoculation }\end{array}$ & $\begin{array}{l}8 \\
\text { Infection } \\
\text { rate }(\%)\end{array}$ & $\begin{array}{l}9 \\
\text { Total }\end{array}$ \\
\hline dsAC1-2 & $0 / 7$ & 0 & $4 / 27(14.8 \%)$ & $0 / 8$ & 0 & $10 / 38(26.3 \%)$ & $0 / 5$ & 0 & $2 / 25(8.0 \%)$ \\
\hline dsAC1-6 & $2 / 7$ & 28.6 & & & & & & & \\
\hline dsAC1-53 & $0 / 7$ & 0 & & & & & & & \\
\hline dsAC1-56 & $2 / 6$ & 33.3 & & & & & & & \\
\hline dsAC1-101 & $0 / 8$ & 0 & $2 / 32(6.2 \%)$ & $0 / 8$ & 0 & & $0 / 8$ & 0 & \\
\hline dsAC1-105 & $2 / 9$ & 22.2 & & $9 / 10$ & 90 & & $2 / 7$ & 28.5 & \\
\hline dsAC1-107 & $0 / 7$ & 0 & & & & & & & \\
\hline dsAC1-152 & $0 / 8$ & 0 & & $1 / 12$ & 8.3 & & $0 / 5$ & 0 & \\
\hline WT (batch 1) & $7 / 14$ & 50 & $7 / 14(50 \%)$ & $11 / 13$ & 84.6 & $11 / 13(84.6 \%)$ & $6 / 9$ & 66.6 & $6 / 9(66.6 \%)$ \\
\hline WT (batch 2) & $11 / 17$ & 64.7 & $11 / 17(64.7 \%)$ & & & & & & \\
\hline
\end{tabular}

Columns 1, 4 and 7 give the number of plants showing mosaic symptoms versus the total number of plants used in the infection test

binding/methionine adenosyltransferase gene (SAMS, EU000298) of cassava.

\section{Small RNA detection and quantification}

Small RNA isolation and detection was performed as described previously. RNA was extracted from leaves of in vitro grown plantlets. Twenty-five $\mu \mathrm{g}$ total RNA was loaded per lane and signal detection was performed with ${ }^{32} \mathrm{P}$-labelled probe using a molecular imager (Bio-Rad Laboratories). The RNA-size markers were prepared as described (Akbergenov et al. 2006) using a pair of DNA oligonucleotides as a template-the T7 promoter oligo 5'-TAATACGACTC ACTATAG- $3^{\prime}$ and the 21 nt oligo $5^{\prime}$-AGTGCTTTAGGTAT GGTTTCCCTATAGTGAGTCGTATTA-3'. Band intensities (counts $/ \mathrm{mm}^{2}$ ) were quantified with Quantity One ${ }^{\circledR}$ software (Bio-Rad Laboratories). Cumulative value of the upper band and the lower band was calculated per lane. The highest cumulative value was set as the $100 \%$ reference. Values of the remaining lanes were expressed as percentage of the reference value. The DNA oligonucleotides used as probes for small RNAs detection are listed in Supplementary Table 1.

\section{Results}

Transgenic cassava transformed with the dsAC1 hairpin cassette are resistant to African cassava mosaic virus

Eight independent transgenic cassava lines were characterized by Southern blot hybridization for the presence and copy number of the transgene (Supplementary Fig. S1). Expression of the transgene was under the control of the
Caulimoflower mosaic virus (CaMV) 35S promoter to produce high levels of hp-derived small interfering RNAs (siRNA) in leaves, where virus transmission by whitefly (Bemisia tabaci) usually occurs. The selected transgenic lines and wild-type plants were bombarded with $350 \mathrm{ng}$ of viral ACMV-NOg DNA per plant. Inoculated plants were kept under greenhouse conditions and symptom severity was determined according to our earlier standard scales (Zhang et al. 2005). In five transgenic lines tested, no mosaic symptom could be observed in the emerging leaves after ACMV bombardment (Fig. 1). The remaining three lines showed reduced infection percentage when compared to the wild-type (WT) cassava (Table 1, columns 1 and 2). Comparison of the infection rate between wild-type plants (group 1: $n=14$, infection rate $=50.0 \%$ /group 2: $n=17$, infection rate $=64.7 \%$, Table 1 , column 3 ) and pooled transgenic plants (group 1: $n=27$, infection rate $=17.4 \%$ group $2: n=32$, infection rate $=6.25 \%$, Table 1, column 3) revealed that the transgenic cassava lines had a significant lower infection rate compared to the WT cassava $(P<0.05$; Pearson chi-square test $)$.

According to our previous work with virus bombardment assays, geminivirus resistance can be broken when the virus load is increased (Zhang et al. 2005). In order to evaluate the efficacy of the RNAi approach in the resistant transgenic cassava lines, we increased the virus load with the biolistic delivery procedure. Three transgenic cassava lines (line dsAC1-2, line dsAC1-101 and line dsAC1-152) having shown an infection rate of $0 \%$ under $350 \mathrm{ng}$ ACMV load were selected for a virus bombardment assay with 700 ng ACMV-NOg DNA load. Line dsAC1-105 that had shown reduced infection rate $(22.2 \%$ of plants infected) in the first resistance screen was included in the second virus bombardment assay. 
Fig. 1 Mosaic symptoms on ACMV-infected WT cassava and ACMV-infected dsAC1-2 cassava line. Plants were biolistically inoculated
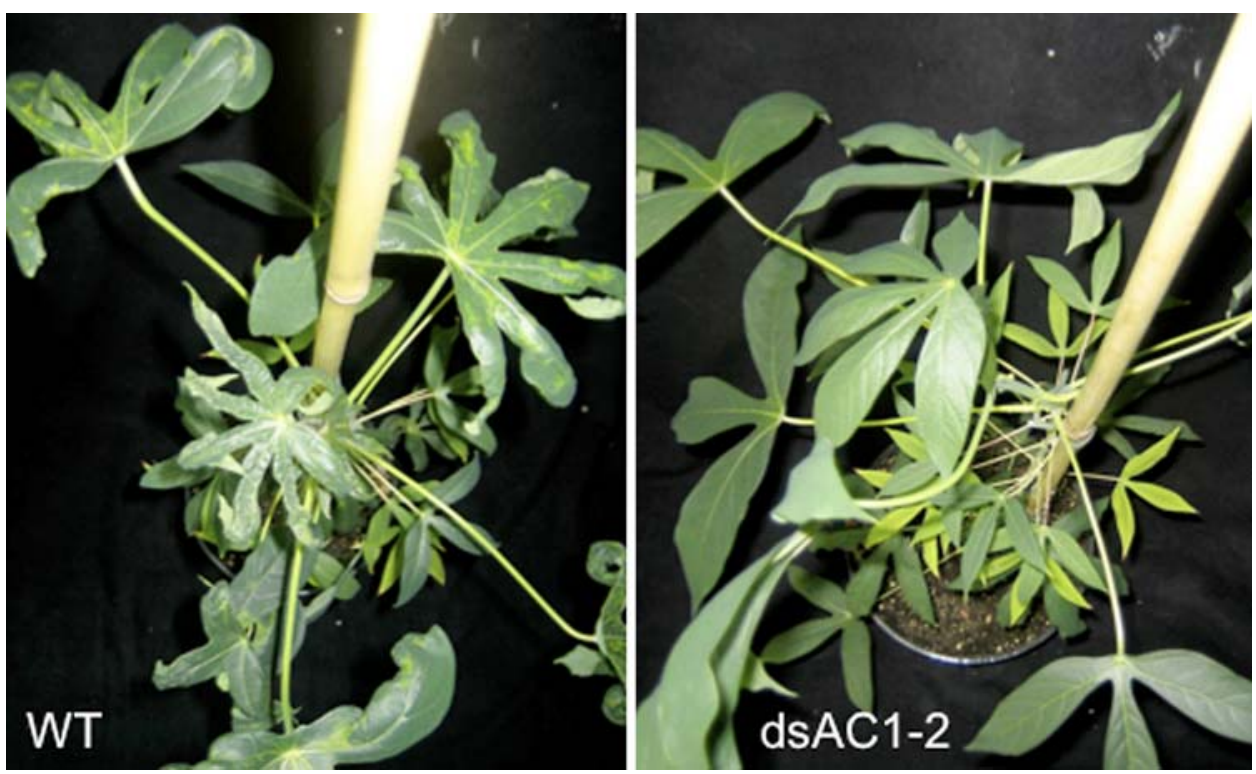

Increasing the viral pressure from 350 to $700 \mathrm{ng}$ brought the infection percentage of the WT cassava from 64.7 to $84.6 \%$ (Table 1, columns 4 and 5). Line dsAC1-2 and line dsAC1-101 showed 0\% ACMV infection under these conditions while line dsAC1-152 had an infection rate of $8 \%$. The infection rate of line dsAC1-105 increased from 22.2 to $90 \%$. The difference between the infection rate of wild-type plants $(n=13$, infection rate $=84.6 \%$, Table 1 , column 6 ) and the infection rate of the pooled transgenic lines $(n=38$, infection rate $=26.3 \%$, Table 1 , column 6 ) was highly significant $(P<0.0001$, Pearson chi-square test), wild-type plants showing a higher infection rate. However, we concluded that an increased viral pressure could break the resistance observed in the first infection assay. Under the $700 \mathrm{ng}$ viral load, line dsAC1-105 reached an infection rate comparable to the WT. Observation of the symptom severity did not reveal significant differences between the WT and the transgenic lines.

In order to evaluate the stability of the resistance under other viral inoculation procedures, we developed an agroinoculation protocol. The resistant lines performed equally well when using the Agrobacterium inoculation method (Table 1, columns 7 and 8). The statistical test confirmed the highly significant difference between the infection rate of the wild-type plants $(n=9$, infection rate $=66.6 \%$, Table 1, column 9) and the infection rate of the pooled transgenic lines $(n=25$, infection rate $=8 \%$, Table 1 , column 9) ( $P<0.001$, Pearson chi-square test), wild-type plants showing a higher infection rate. Semi-quantitative PCR confirmed that leaves of resistant transgenic cassava lines, which developed post-infection, were free of virus DNA (Supplementary Fig. S2).
African cassava mosaic virus resistance correlates with hairpin-derived small RNAs in transgenic cassava lines

It has been hypothesized by Fuentes et al. (2006) that the Tomato yellow leaf curl virus (TYLCV) resistance was due to the high load of hairpin-derived small RNAs in the sole transgenic tomato line that showed resistance. We used a northern blot to obtain relative quantification of the hairpin-derived small RNAs between the selected transgenic cassava lines (Fig. 2). Addition of the $21 \mathrm{nt}$ and the $24 \mathrm{nt}$ signals detected with the Probe 2 fwd gave the highest value for the cassava line 1-152. Using line dsAC1-152 as the reference $(100 \%)$, accumulation of hp-derived siRNA levels in the other transgenic lines could be classified as low (1-15\%; dsAC1-56, dsAC1-105), medium (40\%; dsAC1-6) and high (60-80\%; dsAC1-101, dsAC1-107, dsAC1-2, dsAC1-53). At a virus load of $350 \mathrm{ng} / \mathrm{plant}$, the transgenic lines that showed an infection rate of $0 \%$ in the first ACMV resistance screen (Table 1, columns 1 and 2) were those that had high accumulation of hp-derived small RNAs. Lines with low or medium hairpin-derived small RNAs accumulation had an infection rate varying from 22.2 to $33.3 \%$. When challenged with a very high virus load (700 ng/plant), selected lines with high hp-derived siRNA accumulation maintained an infection rate below $10 \%$ while the wild-type and the transgenic line with low hp-derived siRNA accumulation had infection rate above $80 \%$ (Table 1, columns 4 and 5). The agroinoculation method could also bypass the engineered resistance in the line dsAC1-105 while the transgenic lines with high accumulation showed infection rate of $0 \%$ with this additional inoculation method (Table 1 , columns 7 and 8). We also observed no significant difference between WT and 


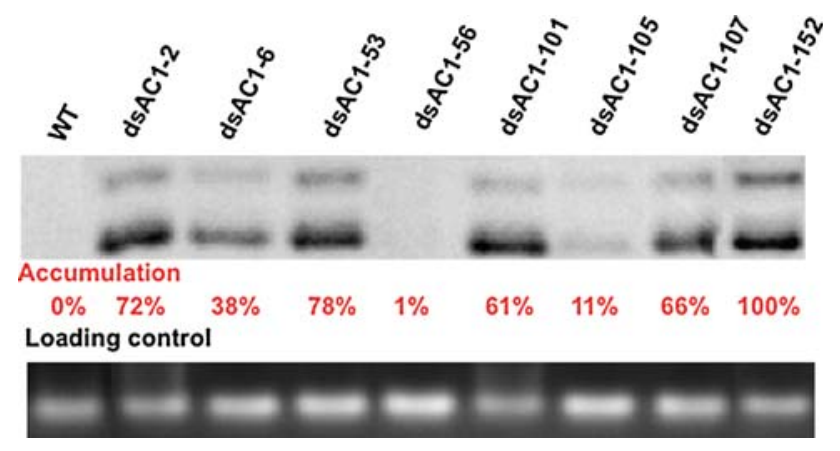

Fig. 2 Small RNA detection and quantification in transgenic cassava plant lines and wild type plant (WT) with ${ }^{32} \mathrm{P}$-labelled dsAC1 probe 1 fwd

line dsAC1-105 in the symptom scores over the first 12 emerging leaves after infection. The recovery phenotype previously observed with the dPro cassava lines (Vanderschuren et al. 2007b) could also not be observed with the infected dsAC1-105 plant. Hairpin-derived small RNAs could be detected over several generations for dsAC1-2 and dsAC1-101 lines (data not shown).

Different classes of small RNAs are generated from the transgene and their pattern depends on their position in the intron hairpin

Because infection rates were negatively correlated with the accumulation of hp-derived siRNAs, we therefore analyzed their origin from the transgene prior to infection. Probe $2 \mathrm{fwd}$ and Probe 2 rev from the center of the Rep/ACl hp region detected three small RNA classes between 21 and 24 nt long (Fig. 3) that were similar to homologous viral sequences we had previously reported (Akbergenov et al. 2006). This pattern of hairpin-derived small RNAs did not seem to be sequence-dependent since a hairpin with ACMV common region (CR) sequence produced the same pattern in cassava using a probe located in the middle of the hairpin (Vanderschuren et al. 2007b). Probes 1 and 3, which span the intron and $3^{\prime} / 5^{\prime}$ end of the hp construct, respectively, revealed significantly different hp-derived siRNA patterns (Fig. 3). The Probe $1 \mathrm{fwd}$, which hybridizes on the $5^{\prime}$ arm of the hairpin transgene, revealed a prominent small RNA class at $20 \mathrm{nt}$ and a weaker one at $21 \mathrm{nt}$. An extra class migrating above the $24 \mathrm{nt}$ band also appeared with the Probe $1 \mathrm{fwd}$. The Probe 1 rev revealed a prominent small RNA class at $22 \mathrm{nt}$. Other classes at $20 \mathrm{nt}$ and $21 \mathrm{nt}$ appeared with a weaker intensity. As for the Probe $1 \mathrm{fwd}$, the Probe $1 \mathrm{rev}$ also revealed an extra band running above the 24 nt class. This latter small RNA class was migrating slightly above the one detected with the Probe $1 \mathrm{fwd}$.

Investigation of the intron end processing showed a striking difference. The intron-spanning Probe 3 fwd and Probe 3 rev (Fig. 3) detected polarity-independent RNAs significantly longer than 24 nt. No difference was observed between the forward and reverse pattern. Probes specific to the intron and the terminator sequences confirmed that the longer-size RNAs were not derived from unspliced or readthrough sequences (data not shown). This suggests that those small RNAs were derived from the base-paired region of the hairpin and that they are homologous to viral sequences. Our results demonstrate that an intron-containing hairpin construct can also generate longer small RNAs. It is currently unknown if the longer small RNAs derived from the hairpin contribute to the interference with virus replication in the resistant lines.

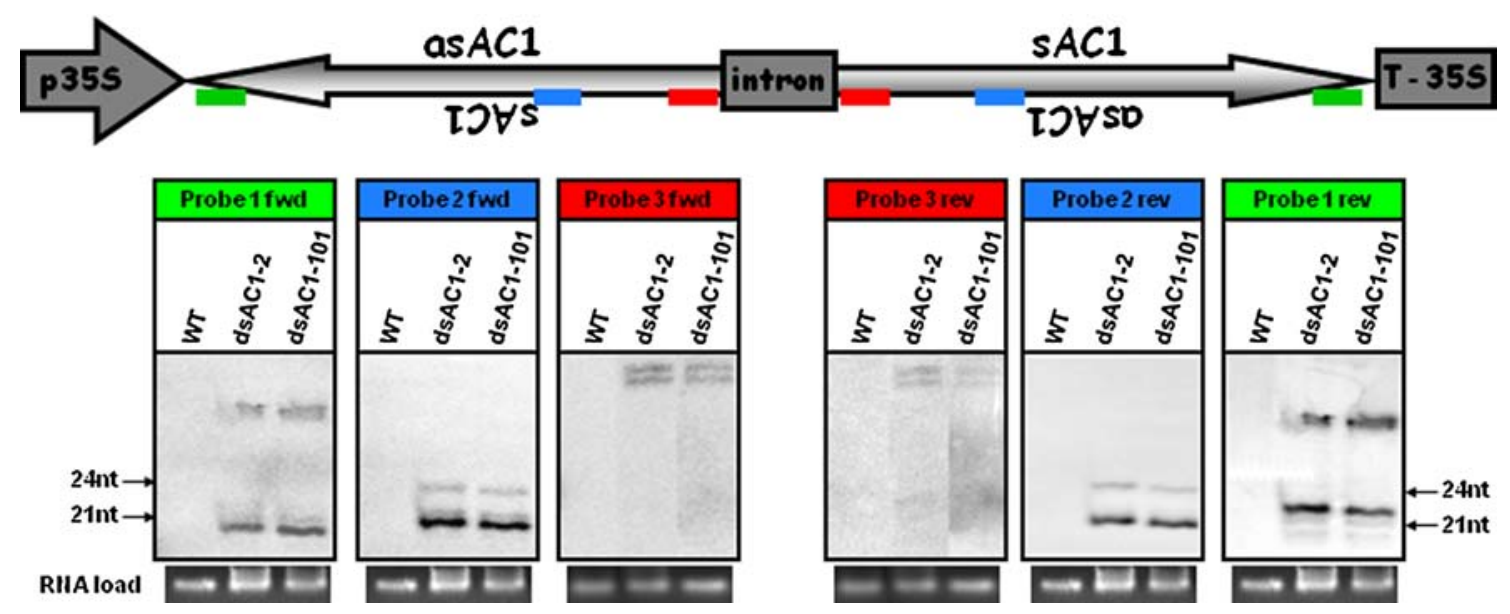

Fig. 3 Scheme of the intron haprin dsAC1 transgene-derived small RNAs detection in transgenic cassava lines using ${ }^{32} \mathrm{P}$-labelled probes located at different positions on the intron-haprin transgene 


\section{Discussion}

Until now, geminivirus resistance has proven to be difficult to engineer in a manner that guarantees trait phenotype for each transgenic line having high level expression of the resistance sequence. This observation seemed independent from the strategy used (i.e. antisense RNA, interfering proteins (CP, AC1), intron hairpin RNA) (Kunik et al. 1994; Hong and Stanley 1996; Frischmuth and Stanley 1998; Zhang et al. 2005; Fuentes et al. 2006; Zrachya et al. 2007; Bonfim et al. 2007; Ribeiro et al. 2007). Our results support the hypothesis that hairpin RNAs can be used to achieve virus resistance in plants. It has an important implication for future strategies to combat CMD in Africa. It raises the possibility to combine several effective short hairpins to target geminivirus species cocktails that plants are exposed to in the field.

The construct that is presented in this study shows several particularities that may explain its efficacy when highly expressed. Its short hairpin length (155 bp) was selected on the basis that complete silencing could be achieved with hairpin length as short as $98 \mathrm{nt}$ (Wesley et al. 2001). We have demonstrated that a correlation exists between the ACMV resistance and the amount of small RNAs generated in the transgenic lines. Several factors can influence the expression level of the hairpin such as the promoter strength, the hairpin length, and the hairpin structure. Introns and their distance to the promoter have been demonstrated to play a role in the gene expression (Rose 2004; Belostotsky and Rose 2005). The combination of a short intron with short hairpin arms and a strong promoter might represent a "winning" strategy to reach sufficient levels of AC1-homologous small RNAs and to consequently render the high-expressing lines able to prevent the virus replication. It is interesting to note that the reported constructions used to produce intron hairpinderived small RNAs against geminiviruses are all harbouring long introns and hairpin arms longer than $400 \mathrm{nt}$ (Fuentes et al. 2006; Zrachya et al. 2007; Bonfim et al. 2007; Ribeiro et al. 2007). Fuentes and colleagues hypothesized the correlation between TYLCV resistance and high level of hairpin-derived small RNAs based on the observation of two transgenic lines. Here we provide further evidence that a positive correlation can be found between geminivirus resistance and hairpin-derived small RNAs in the ACMV-cassava plant system.

The observed dose-dependency can be explained by the importance of the RNA silencing-based antiviral defense pathway and the escalating arms race between viruses and their plant hosts (Waterhouse and Fusaro 2006). In this scheme, small RNAs homologous to viral sequences need to be present in sufficient amounts to ensure complete and efficient silencing of viral mRNAs. The hairpin-derived small RNAs represent an additional barrier to the ones produced by the endogenous silencing machinery. The characterized quantitative effect cannot occult the qualitative effect of the transgene. Our analysis revealed the presence of several small RNAs bands that do not fall in the well-characterized classes (21-24 nt). Small RNAs longer than those resulting from known RNAi-related pathways have already been reported in plants and ciliates (Lee and Collins 2006; Katiyar-Agarwal et al. 2007; Swiezewski et al. 2007). Their role is not yet well characterized but they seem to be involved in silencing for plants or genome rearrangement for ciliates. In plants, longer small RNAs are not exclusive to control of endogenous sequences since virus-homologous sequences have also been detected in infected plants (Hily et al. 2005; Blevins et al. 2006). In the case of the hairpin-derived small RNAs, we observed longer small RNA classes originating only from the $3^{\prime} / 5^{\prime}$ end and the intron end. Our findings show that the hairpin is differentially processed at the hairpin ends. The intron-end derived small RNAs are not likely to be hairpin intermediates since no other classes are detected with the intron-end probe. The $3^{\prime} / 5^{\prime}$ end also showed non-conventional dicer processing pattern since it produced a $20 \mathrm{nt}$ class and one that can be estimated around $30 \mathrm{nt}$.

The efficacy of longer small RNAs for gene silencing, remains to be studied and compared to known RNAirelated small RNAs. If the identified classes prove to be potent silencing effectors, it will force the revision of hairpin design such that the most relevant sequences (i.e. the ones necessary for broad spectrum resistance) are located at the hairpin ends. Several parameters might also be taken in consideration to influence the hairpin-derived small RNAs pattern such as the promoter driving the expression of the hairpin transgene (Wang et al. 2008). On the contrary, inefficacy of longer small RNAs for silencing would require further analysis of the hairpin part that can effectively participate in gene silencing. We are currently addressing this question in the Arabidopsis-Cabbage Leaf Curl Virus system.

Acknowledgements We thank Dr. John Stanley (John Innes Centre) for the ACMV clones, and Drs. Johannes Fütterer (ETH Zurich), Daniel Schöner (ETH Zurich) and Thomas Hohn (University of Basel) for helpful discussions. This work was supported by grants from the Eiselen-Foundation-Ulm and the Bill \& Melinda Gates Foundation Grand Challenges in Global Health Initiative.

\section{References}

Akbergenov R, Si-Ammour A, Blevins T, Amin I, Kutter C, Vanderschuren H, Zhang P, Gruissem W, Meins F Jr, Hohn T, Pooggin MM (2006) Molecular characterization of geminivirus- 
derived small RNAs in different plant species. Nucleic Acids Res 34(2):462-471. doi:10.1093/nar/gkj447

Belostotsky DA, Rose AB (2005) Plant gene expression in the age of systems biology: integrating transcriptional and post-transcriptional events. Trends Plant Sci 10(7):347-353. doi:10.1016/ j.tplants.2005.05.004

Blevins T, Rajeswaran R, Shivaprasad PV, Beknazariants D, SiAmmour A, Park HS, Vazquez F, Robertson D, Meins F Jr, Hohn T, Pooggin MM (2006) Four plant dicers mediate viral small RNA biogenesis and DNA virus induced silencing. Nucleic Acids Res 34(21):6233-6246. doi:10.1093/nar/gk1886

Bonfim K, Faria JC, Nogueira EOPL, Mendes EA, Aragao FJL (2007) RNAi-mediated resistance to Bean golden mosaic virus in genetically engineered common bean (Phaseolus vulgaris). Mol Plant Microbe Interact 20(6):717-726. doi:10.1094/MPMI-20-60717

Boulton MI (2003) Geminiviruses: major threats to world agriculture. Ann Appl Biol 142(2):143. doi:10.1111/j.1744-7348.2003.tb 00239.x

Chellappan P, Masona MV, Vanitharani R, Taylor NJ, Fauquet CM (2004) Broad spectrum resistance to ssDNA viruses associated with transgene-induced gene silencing in cassava. Plant Mol Biol 56(4):601-611. doi:10.1007/s11103-004-0147-9

Fauquet C, Fargette D (1990) African cassava mosaic-virusetiology, epidemiology, and control. Plant Dis 74(6):404-411. doi:10.1094/PD-74-0404

Frischmuth T, Stanley J (1998) Recombination between viral DNA and the transgenic coat protein gene of African cassava mosaic geminivirus. J Gen Virol 79:1265-1271

Fuentes A, Ramos PL, Fiallo E, Callard D, Sanchez Y, Peral R, Rodriguez R, Pujol M (2006) Intron-hairpin RNA derived from replication associated protein $\mathrm{C} 1$ gene confers immunity to Tomato Yellow Leaf Curl Virus infection in transgenic tomato plants. Transgenic Res 15(3):291-304. doi:10.1007/s11248-0055238-0

Hanley-Bowdoin L, Settlage SB, Orozco BM, Nagar S, Robertson D (2000) Geminiviruses: models for plant DNA replication, transcription, and cell cycle regulation. Crit Rev Biochem Mol Biol 35(2):105-140

Hily J-M, Scorza R, Webb K, Ravelonandro M (2005) Accumulation of the long class of siRNAs is associated with resistance to plum pox virus in a transgenic woody perennial plum tree. Mol Plant Microbe Interact 18(8):794-799. doi:10.1094/MPMI-18-0794

Hong Y, Stanley J (1996) Virus resistance in Nicotiana benthamiana conferred by African cassava mosaic virus replication-associated protein (AC1) transgene. Mol Plant Microbe Interact 9(4): 219-225

Kalantidis K, Psaradakis S, Tabler M, Tsagris M (2002) The occurrence of CMV-specific short RNAs in transgenic tobacco expressing virus-derived double-stranded RNA is indicative of resistance to the virus. Mol Plant Microbe Interact 15(8):826833. doi:10.1094/MPMI.2002.15.8.826

Katiyar-Agarwal S, Gao S, Vivian-Smith A, Jin H (2007) A novel class of bacteria-induced small RNAs in Arabidopsis. Genes Dev 21(23):3123-3134. doi:10.1101/gad.1595107

Kunik T, Salomon R, Zamir D, Navot N, Zeidan M, Michelson I, Gafni Y, Czosnek H (1994) Transgenic tomato plants expressing the tomato yellow leaf curl virus capsid protein are resistant to the virus. Biotechnology (NY) 12(5):500-504. doi:10.1038/nbt 0594-500

Lee SR, Collins K (2006) Two classes of endogenous small RNAs in Tetrahymena thermophila. Genes Dev 20(1):28-33. doi:10.1101/ gad.1377006

Legg JP, Fauquet CM (2004) Cassava mosaic geminiviruses in Africa. Plant Mol Biol 56(4):585-599. doi:10.1007/s11103-0041651-7
Legg JP, Owor B, Sseruwagi P, Ndunguru J (2006) Cassava mosaic virus disease in East and central Africa: epidemiology and management of a regional pandemic. Adv Virus Res 67:355418. doi:10.1016/S0065-3527(06)67010-3

Mansoor S, Zafar Y, Briddon RW (2006) Geminivirus disease complexes: the threat is spreading. Trends Plant Sci 11(5):209212. doi:10.1016/j.tplants.2006.03.003

Noris E, Accotto GP, Tavazza R, Brunetti A, Crespi S, Tavazza M (1996) Resistance to tomato yellow leaf curl geminivirus in Nicotiana benthamiana plants transformed with a truncated viral C1 gene. Virology 224(1):130-138. doi:10.1006/viro.1996.0514

Pooggin M, Shivaprasad PV, Veluthambi K, Hohn T (2003) RNAi targeting of DNA virus in plants. Nat Biotechnol 21(2):131-132. doi:10.1038/nbt0203-131b

Ribeiro SG, Lohuis H, Goldbach R, Prins M (2007) Tomato chlorotic mottle virus is a target of RNA silencing but the presence of specific short interfering RNAs does not guarantee resistance in transgenic plants. J Virol 81(4):1563-1573. doi:10.1128/JVI.01238-06

Rose $A B$ (2004) The effect of intron location on intron-mediated enhancement of gene expression in Arabidopsis. Plant J 40(5): 744-751. doi:10.1111/j.1365-313X.2004.02247.x

Shepherd DN, Mangwende T, Martin DP, Bezuidenhout M, Kloppers FJ, Carolissen $\mathrm{CH}$, Monjane AL, Rybicki EP, Thomson JA (2007) Maize streak virus-resistant transgenic maize: a first for Africa. Plant Biotechnol J 5(6):759-767. doi:10.1111/j.14677652.2007.00279.x

Smith NA, Singh SP, Wang MB, Stoutjesdijk PA, Green AG, Waterhouse PM (2000) Gene expression-total silencing by intron-spliced hairpin RNAs. Nature 407(6802):319-320. doi: $10.1038 / 35030305$

Soni R, Murray JA (1994) Isolation of intact DNA and RNA from plant tissues. Anal Biochem 218(2):474-476. doi:10.1006/abio.1994. 1214

Swiezewski S, Crevillen P, Liu F, Ecker JR, Jerzmanowski A, Dean C (2007) Small RNA-mediated chromatin silencing directed to the $3^{\prime}$ region of the Arabidopsis gene encoding the developmental regulator, FLC. Proc Natl Acad Sci USA 104(9):3633-3638. doi: 10.1073/pnas.0611459104

Vanderschuren H, Stupak M, Futterer J, Gruissem W, Zhang P (2007a) Engineering resistance to geminiviruses-review and perspectives. Plant Biotechnol J 5(2):207-220. doi:10.1111/ j.1467-7652.2006.00217.x

Vanderschuren H, Akbergenov R, Pooggin MM, Hohn T, Gruissem W, Zhang P (2007b) Transgenic cassava resistance to African cassava mosaic virus is enhanced by viral DNA-A bidirectional promoter-derived siRNAs. Plant Mol Biol 64(5):549-557. doi: 10.1007/s11103-007-9175-6

Vanitharani R, Chellappan P, Fauquet CM (2003) Short interfering RNA-mediated interference of gene expression and viral DNA accumulation in cultured plant cells. Proc Natl Acad Sci USA 100(16):9632-9636. doi:10.1073/pnas.1733874100

Wang M-B, Abbott DC, Waterhouse PM (2000) A single copy of a virus-derived transgene encoding hairpin RNA gives immunity to barley yellow dwarf virus. Mol Plant Pathol 1:347-356. doi: 10.1046/j.1364-3703.2000.00038.x

Wang MB, Helliwell CA, Wu LM, Waterhouse PM, Peacock WJ, Dennis ES (2008) Hairpin RNAs derived from RNA polymerase II and polymerase III promoter-directed transgenes are processed differently in plants. RNA 14(5):1-11

Waterhouse PM, Fusaro AF (2006) Viruses face a double defense by plant small RNAs. Science 313(5783):54-55. doi:10.1126/science. 1130818

Wesley SV, Helliwell CA, Smith NA, Wang MB, Rouse DT, Liu Q, Gooding PS, Singh SP, Abbott D, Stoutjesdijk PA, Robinson SP, Gleave AP, Green AG, Waterhouse PM (2001) Construct design for efficient, effective and high-throughput gene silencing in 
plants. Plant J 27(6):581-590. doi:10.1046/j.1365-313X.2001. 01105.x

Zhang P, Potrykus I, Puonti-Kaerlas J (2000) Efficient production of transgenic cassava using negative and positive selection. Transgenic Res 9:405-415. doi:10.1023/A:1026509017142

Zhang P, Vanderschuren H, Futterer J, Gruissem W (2005) Resistance to cassava mosaic disease in transgenic cassava expressing antisense RNAs targeting virus replication genes. Plant Biotechnol J 3(4):385-397. doi:10.1111/j.1467-7652.2005.00132.x

Zrachya A, Kumar PP, Ramakrishnan U, Levy Y, Loyter A, Arazi T, Lapidot M, Gafni Y (2007) Production of siRNA targeted against TYLCV coat protein transcripts leads to silencing of its expression and resistance to the virus. Transgenic Res 16(3): 385-398. doi:10.1007/s11248-006-9042-2 